\title{
0139 OUTCOME FROM EMERGENCY SERVICES FOR EMERGENCY INJURY BY ADVANCE TEAM, EMERGENCY MEDICAL SERVICE, KHON KAEN HOSPITAL
}

T Piyawatchwela* Correspondence: Trauma center and critical care, Khon Kaen Regional Hospital; Khon Kaen province, Thailand 40000, Thailand

\subsection{6/ip.2010.029215.139}

An effective emergency medical service system (EMSS) which could save lives of those who got injured from accidents or critical illness requited an assessment and rapid and appropriate care for each individuals. Patients survival rate during the transportation from the scene to the hospital has also been considered as one of qualitative indexes for EMSS.

Objective To evaluate an effectiveness of services for patients from accidents and in critical illness at the scene of the Advance team, EMS, Khon Kaen hospital during the year 2008.

Methods Retrospective study from EMS report, Khon Kaen hospital.

Subjects Patients from accidents or severely critical illness received service from EMS, Khon Kaen hospital at the scene during October 1, 2007 to September 30, 2008. Data were descriptively analysed as percentage.

Results Total subjects of 191 patients (122 males and 69 females) were categorised as 140 critically ill patients $(73.30 \%)$ and 51 patients from accidents $(26.70 \%)$. One hundred and thirty patients $(72.77 \%)$ were treated in the Medical Unit, followed by patients with road accidents (38 patients; 19.89\%). Numbers of patients initially evaluated as unconscious with GCS score 3 and those who required CPR at the scene and later brought to the hospital were $73(38.22 \%)$ and 35 (18.32\%), respectively. For patients who were brought to the hospital, 20 patients $(57.14 \%)$ died at the emergency room while 15 patients (42.68\%) survived. Average response times for patients from accidents and patients with other critical illnesses were 4.40 min and $6.54 \mathrm{~min}$, respectively.

Conclusion It was found from this study that all related personnel must work precisely and quickly. These are considered important factors regarding and effective medical service to save patients lives. 